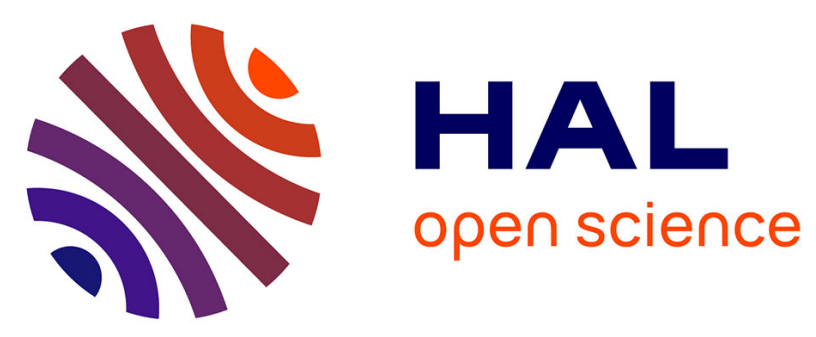

\title{
Cooperative Localization with Reliable Confidence Domains between Vehicles sharing GNSS Pseudoranges Errors with no Base Station
}

Khaoula Lassoued, Philippe Bonnifait, Isabelle Fantoni

\section{- To cite this version:}

Khaoula Lassoued, Philippe Bonnifait, Isabelle Fantoni. Cooperative Localization with Reliable Confidence Domains between Vehicles sharing GNSS Pseudoranges Errors with no Base Station. IEEE Intelligent Transportation Systems Magazine, 2017, 9 (1), pp.22-34. 10.1109/MITS.2016.2630586 . hal-01390329

\section{HAL Id: hal-01390329 \\ https://hal.science/hal-01390329}

Submitted on 1 Nov 2016

HAL is a multi-disciplinary open access archive for the deposit and dissemination of scientific research documents, whether they are published or not. The documents may come from teaching and research institutions in France or abroad, or from public or private research centers.
L'archive ouverte pluridisciplinaire HAL, est destinée au dépôt et à la diffusion de documents scientifiques de niveau recherche, publiés ou non, émanant des établissements d'enseignement et de recherche français ou étrangers, des laboratoires publics ou privés. 


\title{
Cooperative Localization with Reliable Confidence Domains between Vehicles sharing GNSS Pseudoranges Errors with no Base Station
}

\author{
Khaoula Lassoued, Philippe Bonnifait and Isabelle Fantoni
}

\begin{abstract}
Cooperation between road vehicles through information exchange is a promising way to enhance their absolute and relative positions. This paper presents an approach for generating, sharing and applying Global Navigation Satellite System (GNSS) pseudorange corrections through a V2X communication network. Conventionally, differential corrections are generated by fixed base stations with known positions and sent to mobile users. Here, the proposed cooperative method has no central server and the estimation of the raw measurements errors is done in a fully distributed way. Using a model of the correlation of the pseudorange errors and through the knowledge of the local motions of the vehicles obtained by Dead Reckoning (DR) or tracking, a non linear observability shows that the estimation problem is solvable. A cooperative and fully distributed estimation method is then presented using Set Inversion and Constraint propagation techniques. Positions, pseudorange estimated errors and DR data are shared in the network of vehicles and confidence is handled by intervals, in a bounded error context. This allows computing highly reliable confidence domains with no direct range measurements, which is crucial for applications involving close proximity navigation. Indeed, the proposed data fusion framework does not require any linearization of the equations and is insensitive to the data incest problem since the same information can be exploited several times in the computation process without making the estimation overconverge. Results using real measurements are presented to illustrate the performance of the proposed cooperative method in comparison with standalone estimation. A classical sequential Bayesian method has also been implemented on the same data set and compared in terms of accuracy and confidence with a ground truth system.
\end{abstract}

\section{INTRODUCTION}

To make road vehicles cooperate efficiently via V2X communications, position estimates with reliable confidence indicators are crucial. Mutual cooperation through information exchange is a promising way to enhance positioning accuracy and reduce uncertainty arising from the use of low cost sensors. For example, mono-frequency Global Navigation Satellite Systems (GNSS) receivers in complex environments usually leads to offsets between real and observed positions (Fig. 1). These systematic offsets (i.e, biases) are often due to inaccurate satellite positions, atmospheric and tropospheric errors. The impact of these biases on vehicles localization should not be neglected. Cooperation and exchange of biases estimates between vehicles can reduce significantly these systematic errors. However, distributed cooperative localization based on sharing estimates is subject to data incest problems (i.e, reuse of identical information in the fusion process) that often lead to over-convergence problems [1], [2]. When position information is used in a safety-critical context (e.g. autonomous vehicles navigation in proximity), one should guaranty the consistency of the localization estimates. In this context, we mainly aim to improve the absolute and relative performances of vehicles localization through cooperation. Moreover, we focus on characterizing reliable confidence domains (see Fig. 1) that contain vehicles positions with high reliability.

Common approaches for localization are Bayesian methods relying on Extended Kalman Filter (EKF) or Unscented Kalman Filter since in practice we face nonlinear systems [1]. EKF-based methods have

The authors are with Sorbonne Universités, Université de Technologie de Compiègne, CNRS UMR 7253 Heudiasyc, 60200 Compiègne, France.

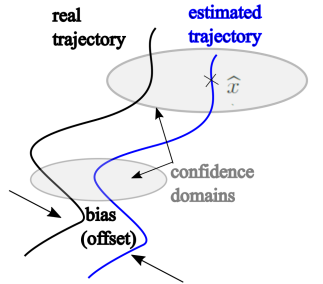

FIGURE 1: Illustration of the effects of biased measurements. Confidence domains are zones in which the vehicle is highly located.

been used for many years and remain the main tool for nonlinear estimation in problems ranging from missile guidance to GNSS positioning. The efficacy of these techniques to provide accurate and consistent estimates depends greatly on the fidelity of the system and sensor models. Furthermore, an EKF is not guaranteed to converge when the initial estimate of the state is wrong, or when the process is modeled incorrectly owing to its linearization. Moreover, it is sensitive to tuning [3]. It has been suggested that, instead of assuming noise sources to follow known probabilistic distributions, only bounds of the errors can be managed. These methods are known as setmembership or bounded-error estimation. Set-membership methods are considered as efficient solutions to data incest problem. This kind of approach enables merging adequately the information even when it is reused several times. It also provides reliable domains. Moreover, the use of non-linear models does not require any linearization [4]. In this work, Set Inversion Via Interval Analysis (SIVIA) is used as the core methodology. It is a particular set-membership method that relies on interval analysis. Often, SIVIA is used in order to characterize guaranteed solution sets of non-linear problems by bisecting recursively a searching space [5]. It has been successfully applied for model parameters estimation in [6]. When reliable robots pose confidence domains are required, the authors of [7] and [8] have shown that set inversion can manage very well the uncertainty. However, SIVIA alone is not suitable for real time, especially when the dimension of the space is high. A strong improvement is to use SIVIA while simultaneously solving a Constraint Satisfaction Problem (CSP) in order to limit at the maximum the number of bisections [9]. Recently, several studies have used these new set inversion methods for localizing multiple robots. Bethencourt [10] used distributed set-membership algorithms in a group of AUVs to accomplish a swarm cooperative mission based on inter-temporal measurements. Cooperative localization using multi-GNSS receivers and V2V/V2I communications is an increasing domain as shown by Bento et al. [11]. In [12], the authors have studied a cooperative localization method for multiple ground robots based on constraint propagation techniques. However, all these works did not consider biased measurements. However, there are some works based on Bayesian methods that consider biased measurements. In [13], a cooperative tightly-coupled relative GPS/INS integration is presented using simulated data of two vehicles. This decentralized cooperative approach aims to enhance vehicles positions by correcting pseudoranges biases and receivers clocks biases when using pseudoranges double differences techniques. More recently, Mattern et al. have 


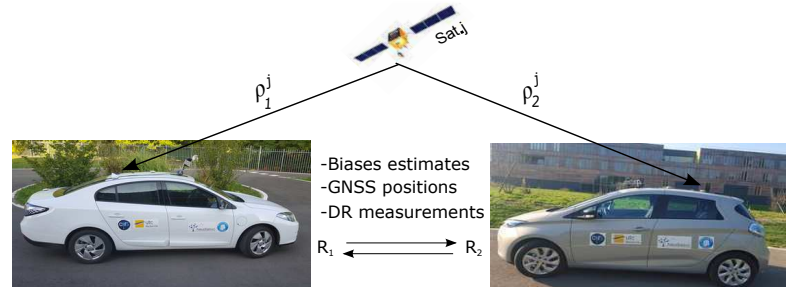

FIGURE 2: Experimental vehicles and shared data

extended this previous work to handle real world data problems. They have proposed a cooperative algorithm based on UKF filtering to improve the absolute and relative localization of six vehicles, when using GPS data and digital maps [14].

In this paper, we propose a new formulation of multi-vehicles cooperation using set inversion with CSP techniques on intervals. When using V2X communications, vehicles cooperate and exchange information such that each vehicle can compute the positions of the partners with a reliable domain. The ego-motion of every vehicle can also be used to improve the cooperation. The proposed method relies on the exchange of positions estimates, pseudorange estimated errors and DR data (see Fig. 2). A first contribution of this paper is to show that it is possible to improve the ego positioning and mutual localization between vehicles by sharing GNSS biased pseudoranges using a model of the correlation of the pseudorange errors and through the knowledge of the local motions of the vehicles done by DR or tracking. This represents the objective of section II, where the system modeling and an observability study of the cooperative system are introduced. Then, in section III, the proposed distributed estimation method is presented. It allows the data fusion of the estimated biases in a distributed way with no central fusion node and with no base station. An experimental evaluation with two vehicles is performed in section IV to evaluate the performance in real conditions using a ground truth system. The performance of the cooperation is clearly highlighted. A classical sequential Bayesian method has also been implemented on the same data set and is compared in terms of accuracy and confidence. This comparison also represents a contribution of this paper.

\section{SYSTEM MODELING AND OBSERVABILITY ANALYSIS}

The data fusion of GNSS measurements with DR measurements is an approach often adopted to enhance localization accuracy [15]. In this section, the mathematical models of the measurements are described and an observability study of the cooperative system is considered. The observability study is an essential step in vehicles localization context. One should check if the vehicles positions and measurements biases can be determined before the development of any observer.

\section{A. Models}

The GNSS raw measurements considered here are $L 1$ pseudoranges. The pseudorange $\rho_{i}^{j}$ [16] is the measure of each vehicle $R_{i}(i=$ $\left.\left\{1, \ldots, n_{r}\right\}\right)$ located at coordinates $\boldsymbol{p}_{i}=\left[x_{i}, y_{i}, z_{i}\right]$ to each satellite $j\left(j=1, \ldots, n_{s}\right)$ at position $\boldsymbol{p}^{j}=\left[x^{j}, y^{j}, z^{j}\right]$. We model the common GNSS error $b^{j}$ as additive errors on pseudorange. The model of $\rho_{i}^{j}$ is expressed in Eq. (1). Please refer to [17] for further details.

$$
\rho_{i}^{j}=\sqrt{\left(x_{i}-x_{j}\right)^{2}+\left(y_{i}-y_{j}\right)^{2}+\left(z_{i}-z_{j}\right)^{2}}+b^{j}+d_{i}+\beta^{j}
$$

where $d_{i}$ represents the receiver clock offset and $\beta^{j}$ the measurement noise.
Let ${ }^{i} \boldsymbol{y} \in \mathbb{R}^{n_{s}}$ be the vector of $n_{s}$ pseudoranges measurements and ${ }^{i} \boldsymbol{x} \in \mathbb{R}^{n}$ be the state vector of vehicle $i$. The observation model at discrete time $k$ is defined as follows

$$
{ }^{i} \boldsymbol{y}_{k}=\boldsymbol{g}\left({ }^{i} \boldsymbol{x}_{k}\right)
$$

Suppose that each vehicle is equipped with a GNSS receiver and DR sensors that provide speed and heading in an input vector ${ }^{i} \boldsymbol{u}(t)=\left[\begin{array}{ll}v_{i} & \psi_{i}\end{array}\right]^{T}$. A 2D unicycle evolution model for the pose components, a linear model for the receiver clock offset and an autoregressive (AR) model for the pseudoranges errors are concatenated and described by a continuous function $f$ in a local East North Up frame (time $t$ is omitted for clarity):

$$
\boldsymbol{f}\left({ }^{i} \boldsymbol{x},{ }^{i} \boldsymbol{u}\right)=\left\{\begin{array}{l}
\dot{x}_{i}=v_{i} \cos \left(\psi_{i}\right) ; \quad \dot{y}_{i}=v_{i} \sin \left(\psi_{i}\right) ; \quad \dot{z}_{i}=0 \\
\dot{d}_{i}=d r_{i} ; \quad \dot{d r_{i}}=0 ; \quad \dot{b}^{j}=a b^{j}
\end{array}\right.
$$

$d_{i}$ and $d r_{i}$ are respectively the receiver clock offset and its drift to be estimated. The AR parameter $a=e^{-T e / \tau}, T_{e}$ being the sampling time and $\tau$ the time constant of the model bias $b^{j}(a=0.9995$, $\tau=6.2 \mathrm{~min}$ ). The indexes of the vehicles and common satellites are respectively denoted by $i \in\left\{1 \ldots n_{r}\right\}$ and $j \in\left\{1 \ldots n_{s}\right\}$.

\section{B. Observability analysis}

Errors on pseudorange measurements are spatially correlated and similar for nearby users [16] but not directly observed with no base station. Therefore, it is essential to study the observability to evaluate if the problem is solvable. Rife and Xiao [18] have shown that it is not possible to estimate biases simply by sharing GNSS pseudorange measurements between vehicles communicating via a Vehicle-to-Vehicle (V2V) network in a snapshot way (Epoch by Epoch). They highlighted the limitation of distributing only GNSS data and proposed to add georeferenced information by using camera-based lane boundary sensor. A natural question that arises is whether GNSS biases are observable when adding vehicles motion information and errors evolution model.

In this section, we investigate the observability of the cooperative localization problem of vehicles sharing biases estimates when they are moving. The cooperative system described in section II-A given $\mathrm{DR}$ and pseudorange measurements is nonlinear. Therefore, we use the observability rank criterion based on Lie-Derivatives [19] to determine the conditions under which the system is locally weakly observable. Note that Martinelli and Siegwart [20] have employed this criterion to investigate the observability of $2 \mathrm{D}$ cooperative localization of mobile robots. Recently, Zhou et al. [21] have used the Lie derivatives to determine the conditions for the observability of $2 \mathrm{D}$ relative pose of pairs of mobile robots using range measurements. In the sequel, a test of Lie derivatives is considered for vehicles sharing GNSS errors. This study is inspired by the work of Zhou et al. [21].

Definition 1. (Observability Rank Condition): The observability rank condition is satisfied when the observability matrix is full rank.

Theorem 1. (Observability Sufficient Condition): If the system satisfies the observability rank condition at a given state $\boldsymbol{x}^{0}$ (at some time), then the system is locally weakly observable at $\boldsymbol{x}^{0}$ [19].

Let consider $n_{r}$ vehicles and $n_{s}$ common visible satellites. Let $\hat{\boldsymbol{x}}$ be the estimated state of the cooperative system (S) as follows

$$
\hat{\boldsymbol{x}}=\left[\hat{\boldsymbol{p}}_{1}, \hat{d}_{1}, \ldots, \hat{\boldsymbol{p}}_{n_{r}}, \hat{d}_{n_{r}}, \hat{b}^{1}, \ldots, \hat{b}^{n_{s}}\right]^{T}
$$


with $\operatorname{dim}(\hat{\boldsymbol{x}})=4 n_{r}+n_{s} . \hat{\boldsymbol{p}}_{1 \cdots n_{r}}$ are the $3 \mathrm{D}$ vehicles positions, $\hat{d}_{1 \cdots n_{r}}$ represent the receivers clock offsets. $\left(\hat{b}^{1 \cdots n_{s}}\right)$ denote the biases on $n_{s}$ common pseudorange measurements between vehicles.

The considered evolution model in this study consists in the first four DR equations of the system (3) and the last equation for the evolution of biases.

Let consider $u=\left[v_{1} \ldots v_{n_{r}}\right]^{T}$ the input of the system (S) in (4). The nonlinear DR model of (S) can be written as follows

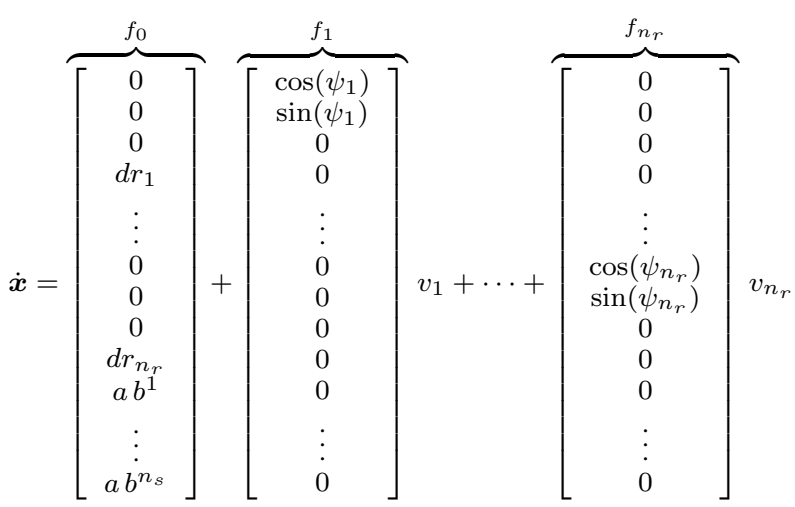

The nonlinear observation equations are given by

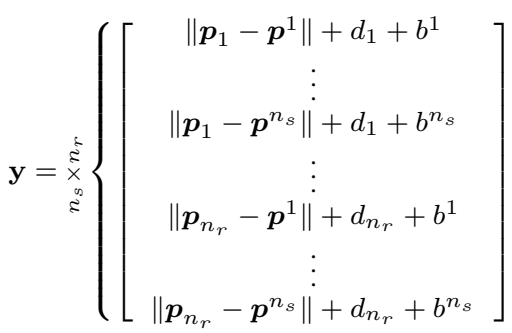

We compute hereafter the necessary Lie derivatives of $\mathbf{y}$ and their gradients:

Zeroth-order Lie derivatives $\left(\mathcal{L}^{0} \mathrm{y}\right)$ :

$$
\mathcal{L}^{0} \mathbf{y}=\mathbf{y}
$$

with gradient:

$$
\begin{aligned}
& \nabla \mathcal{L}^{0} \mathbf{y}=j \operatorname{acobian}(\mathbf{y})
\end{aligned}
$$

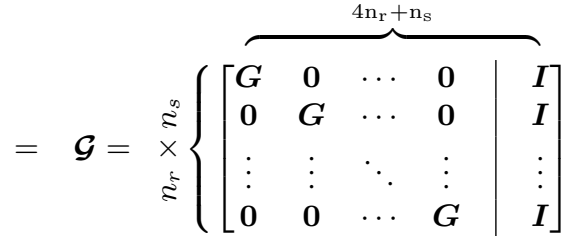

where $\boldsymbol{I}$ is the identity matrix with $\operatorname{dim}(\boldsymbol{I})=n_{s} \times n_{s}$ and $\mathcal{G}$ is the geometry matrix described in [18] where $\boldsymbol{G}$ is defined as follows:

$$
\boldsymbol{G}=\left[\begin{array}{cc}
\left(\boldsymbol{u}^{1}\right)^{T} & 1 \\
\left(\boldsymbol{u}^{2}\right)^{T} & 1 \\
\vdots & \vdots \\
\left(\boldsymbol{u}^{n_{s}}\right)^{T} & 1
\end{array}\right]
$$

with $\operatorname{dim}\left(\boldsymbol{G}_{i}\right)=n_{s} \times 4$, the unit vector $\boldsymbol{u}^{j}$ in $\boldsymbol{G}$ is the estimated line of sight from the satellite $j$ to each user receiver $i$. This pointing vector is the same for all users when they are assumed to be in close proximity (i.e. distance between vehicles $\leq 10 \mathrm{~km}$ ):

$$
\boldsymbol{u}^{j}=\left(\boldsymbol{p}_{i}-\boldsymbol{p}^{j}\right) /\left\|\boldsymbol{p}_{i}-\boldsymbol{p}^{j}\right\|
$$

First-order Lie derivatives $\left(\mathcal{L}_{f_{0}}^{1} \mathrm{y}\right)$ :

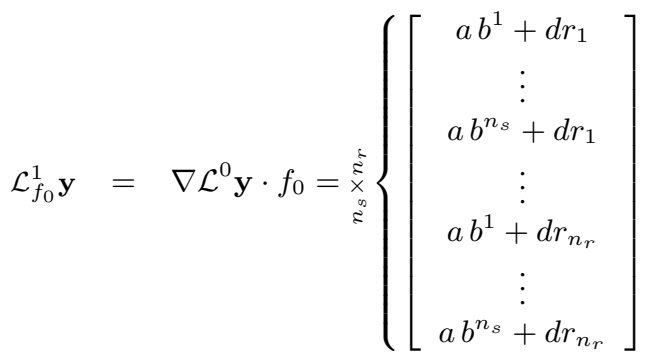

with gradient:

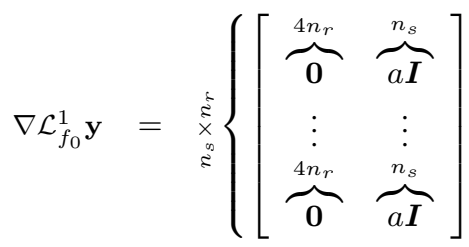

The observability matrix is now:

$$
\mathcal{O}=\left[\begin{array}{c}
\nabla \mathcal{L}^{0} \mathbf{y} \\
\nabla \mathcal{L}_{f_{0}}^{1} \mathbf{y}
\end{array}\right] .
$$

The role of the matrix in Eq. (10) in the observability analysis of a nonlinear system is given in [19], and recalled in definition 1 and theorem 1.

Below, we compute the rank of the observability matrix (10) and determine the necessary conditions under which the system (S) can be locally weakly observable. Here, we have:

$$
\operatorname{rank}(\mathcal{O})=\operatorname{rank}\left(\nabla \mathcal{L}^{0} \mathbf{y}\right)+\operatorname{rank}\left(\nabla \mathcal{L}_{f_{0}}^{1} \mathbf{y}\right) .
$$

The rank of $\nabla \mathcal{L}^{0} \mathbf{y}$ has been studied in [18]. They proved that $\operatorname{rank}\left(\nabla \mathcal{L}^{0} \mathbf{y}\right)=4\left(n_{r}-1\right)+n_{s}$. It is straightforward to determine the rank of $\nabla \mathcal{L}_{f_{0}}^{1} \mathbf{y}$. Since the number of linearly independent equations in $\nabla \mathcal{L}_{f_{0}}^{1} \mathbf{y}$ appears to be $n_{s}$ (i.e $\left.\operatorname{rank}\left(\nabla \mathcal{L}_{f_{0}}^{1} \mathbf{y}\right)=n_{s}\right)$ if we have $a \neq 0$ (i.e. auto-regressive model of the biases). So, according to (11) we get:

$$
\operatorname{rank}(\mathcal{O})=4\left(n_{r}-1\right)+2 n_{s}
$$

In order to get a full rank of $\mathcal{O}$, one must discuss the least required number of $n_{s}$ common satellites between users. It is obvious that $\operatorname{rank}(\mathcal{O})$ can not exceed the $n_{I}$ unknowns states of $(\mathrm{S})$ which is equal to $4 n_{r}+n_{s}$ (i.e. $\operatorname{rank}(\mathcal{O}) \leq 4 n_{r}+n_{s}$ ), so to get a full rank of $\mathcal{O}$ one must determine $n_{s}$ such that

$$
\operatorname{rank}(\mathcal{O}) \geq 4 n_{r}+n_{s}
$$

By replacing (12) in (13) we get: $n_{s} \geq 4$. The observability rank condition (definition 1) is obtained when this condition is satisfied. According to theorem 1, it can be concluded that the system is locally 


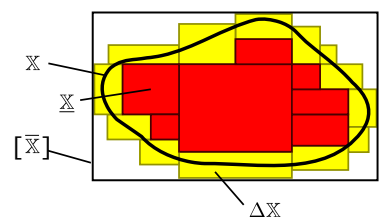

Figure 3: Bracketing of the solution set $\mathbb{X}$ between two subpavings $\mathbb{X}$ and $\overline{\mathbb{X}}=\underline{\mathbb{X}} \cup \Delta \mathbb{X} .[\overline{\mathbb{X}}]$ is the hull box of $\overline{\mathbb{X}}$.

weakly observable regardless the number of users $\left(n_{r}\right)$ if the biases have an auto-regressive behavior and at least 4 common satellites between the vehicles. Please note also that, as we have only used the Lie derivative with respect to $f_{0}$, the system is observable even if the the vehicles are motionless.

\section{Distributed Estimation With SET INVERSion to COMPute Reliable Confidence Domains}

\section{A. Set Inversion with Constraints Propagation for State Estimation}

To perform a state estimation in a bounded error framework with intervals, one needs solving a set inversion problem. The objective is to determine the unknown state $\mathbb{X} \subset \mathbb{R}^{n}$ such as $\mathbf{f}(\mathbb{X}) \subset \mathbb{Y}$, where $\mathbb{Y}$ is the known set of measurements. The objective is to compute the reciprocal image $\mathbb{X}=\mathbf{f}^{-1}(\mathbb{Y})$. A guaranteed approximation of the solution set $\mathbb{X}$ can be done using 2 sub-pavings which bracket the solution set as follows: $\mathbb{X} \subset \mathbb{X} \subset \overline{\mathbb{X}}$ (Fig. 3).

A box $[\mathbf{x}]$ of $\mathbb{R}^{n}$ is feasible if it is inside $\mathbb{X}$ and unfeasible if it is outside $\mathbb{X}$, otherwise $[\mathbf{x}]$ is indeterminate. By using an inclusion function $[\mathbf{f}]$ of function $f$, one can identify the feasibility of the boxes using the following tests:

- If $[\mathbf{f}]([\mathbf{x}]) \subset \mathbb{Y}$ then $[\mathbf{x}]$ is feasible

- If $[\mathbf{f}]([\mathbf{x}]) \cap \mathbb{Y}=\emptyset$ then $[\mathbf{x}]$ is unfeasible

- Else $[\mathbf{x}]$ is indeterminate.

SIVIA solves the set inversion problem by testing recursively the feasibility of candidate boxes, starting from an arbitrarily large initial box $\left[\mathbf{x}_{\mathbf{0}}\right]$ [6]. If a box is feasible, it is stored in the inner solution set $\mathbb{X}$. If a box is unfeasible, it is discarded since the resulting box $([\mathbf{f}]([\mathbf{x}]))$ is outside the measurement domain $\mathbb{Y}$. Finally, an indeterminate box is bisected into two sub-boxes and stored in a list $\mathfrak{L}$ waiting to be treated.

In high dimension, SIVIA is not suitable for real time due to the computation time of the bisections. One solution is to use "SIVIAP", a SIVIA with Constraints Propagation (CP) on intervals. CP is very efficient in terms of computation and reduces the number of needed bisections by contracting boxes in order to speed up the processing. SIVIAP involves the formalization of a Constraint Satisfaction Problem. A CSP is denoted by $\mathcal{H}$ (Eq.14) and provides the box $[x]$ that satisfies all the constraints $F$.

$$
C S P:\{\mathcal{H}:(F(x)=\mathbf{0} \mid x \in[x])\}
$$

Contracting $\mathcal{H}$ means replacing $[\mathbf{x}]$ by a smaller domain $\left[\mathbf{x}^{\prime}\right]$ such that the solution set remains unchanged. The contractors used in this work are Forward-Backward Propagation and Waltz algorithm [22] intersection. More details can be found in [23].

\section{B. Cooperative Localization Problem Statement}

Vehicles share their estimated GNSS errors, their DR measurements and their positions. The objective is to get estimates with reliable confidence domains that contain, with high probability, the true positions of the vehicles with little pessimism.

Let us describe the variables, the domains and the constraints of the considered CSP.

\section{Variables}

In each agent $R_{i}$, there is an ego state to be estimated and a tracked position of every known other vehicle $R_{o}$ in the group, where $o \in$ $\left\{1, \ldots, n_{r}-1\right\}$ and $o \neq 0$.

Ego state: let $\nu=\left\{x_{i}, y_{i}, z_{i}, b^{1}, \ldots, b^{n_{s}}, d_{i}, d r_{i}\right\}$ be the variables of the ego state ${ }^{i} \boldsymbol{x}_{\text {ego }}$ of $R_{i}$ of dimension $n=5+n_{s}$. Only these variables are considered in the CSP. Let denote the absolute position by ${ }^{i} \boldsymbol{q}=\left\{x_{i}, y_{i}, z_{i}\right\}$, the biases of all $n_{s}$ satellites in view by ${ }^{i} \boldsymbol{b}=$ $\left\{b^{1}, \ldots, b^{n_{s}}\right\}$ and the inner variables of $R_{i}$ by ${ }^{i} \boldsymbol{\xi}=\left\{d_{i}, d r_{i}\right\}$ which contain respectively the clock offset $d_{i}$ and its drift $d r_{i}$.

Tracked position: let ${ }^{i} \boldsymbol{q}_{o}=\left\{{ }^{i} \boldsymbol{q}_{1}, \ldots,{ }^{i} \boldsymbol{q}_{n_{r}-1}\right\}$ be the positions of the other vehicles estimated by agent $R_{i}$.

The ego input of $R_{i}$ is denoted by ${ }^{i} \boldsymbol{u}_{\text {ego }}=\left\{v_{i}, \psi_{i}\right\}$, where $v_{i}$ and $\psi_{i}$ are respectively the linear speed and the heading angle. ${ }^{i} \boldsymbol{u}_{o}=$ $\left\{{ }^{i} \boldsymbol{u}_{1}, \ldots,{ }^{i} \boldsymbol{u}_{n_{r}-1}\right\}$ represents the input of $R_{o}$ composed of ${ }^{i} \boldsymbol{u}_{o}=$ $\left\{v_{o}, \psi_{o}\right\}$. This information is received from the others.

\section{Domains}

The domains of the variables are sets which enclose the true value of the variables and are represented by boxes, i.e. vectors of intervals of

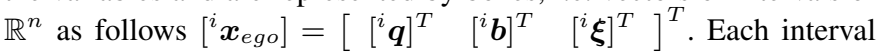
contains the unknown variable $[\nu]=\left[\nu_{\text {true }}-\delta_{\nu}, \nu_{\text {true }}+\delta_{\nu}\right]$, where $\nu_{\text {true }}$ represents the true value of $\nu$ and $\delta_{\nu}$ is the bound of the error.

\section{Constraints}

The constraints that link the variables at each time $k$ are the evolution and observation models:

$$
(i){ }^{i} \boldsymbol{x}_{\text {ego }, k}=\boldsymbol{f}_{k}\left({ }^{i} \boldsymbol{x}_{\text {ego }, k-1},{ }^{i} \boldsymbol{u}_{\text {ego }}\right)
$$

$$
\left(\text { ii) }{ }^{i} \boldsymbol{y}_{k}=\boldsymbol{g}\left({ }^{i} \boldsymbol{x}_{\text {ego }, k}\right)\right.
$$

The constraint (i) corresponds to the dynamic equation of the model, while the constraint (ii) defines the CSP which is used in SIVIAP: ${ }^{i} \boldsymbol{y}_{k}$ are the pseudoranges set to be inverted and ${ }^{i} \boldsymbol{x}_{e g o, k}$ is a prior feasible box coming from a prediction stage.

\section{Solver}

SIVIAP approximates the state vector ${ }^{i} \boldsymbol{x}_{\text {ego,k }}$ such that $\boldsymbol{g}\left({ }^{i} \boldsymbol{x}_{\text {ego }, k}\right) \subseteq$ $\left[{ }^{i} \boldsymbol{y}_{k}\right]$ using a forward-backward contractor. The set to be characterized by SIVIAP is:

$$
\begin{aligned}
C S P & =\left\{{ }^{i} \boldsymbol{x}_{\text {ego }, k} \subseteq\left[{ }^{i} \boldsymbol{x}_{\text {ego }, k}\right] \backslash \boldsymbol{g}\left({ }^{i} \boldsymbol{x}_{\text {ego }, k}\right) \subseteq\left[{ }^{i} \boldsymbol{y}_{k}\right]\right\} \\
& =\boldsymbol{g}^{-1}\left(\left[{ }^{i} \boldsymbol{y}_{k}\right]\right) \cap\left[{ }^{i} \boldsymbol{x}_{\text {ego }, k}\right]
\end{aligned}
$$

\section{Distributed algorithm}

The same algorithm (1) runs in every vehicle $R_{i}$. Agent $R_{i}$ predicts its ego state ${ }^{i} \boldsymbol{x}_{\text {ego }}$ using the evolution model and its DR inputs $\left(v_{i}, \psi_{i}\right)$ measured at high frequency (line 1). Moreover, it tracks the other vehicles (line 2) using their last received DR inputs.

Lines $(4 \cdots 14)$ of the algorithm consist in updating the predicted state ${ }^{i} \boldsymbol{x}_{\text {ego }}$ with respect to the GNSS measurements which are available every $0.2 s$. In order to reduce the outliers at each time $k$ when the GNSS measurements are available, a validation process 


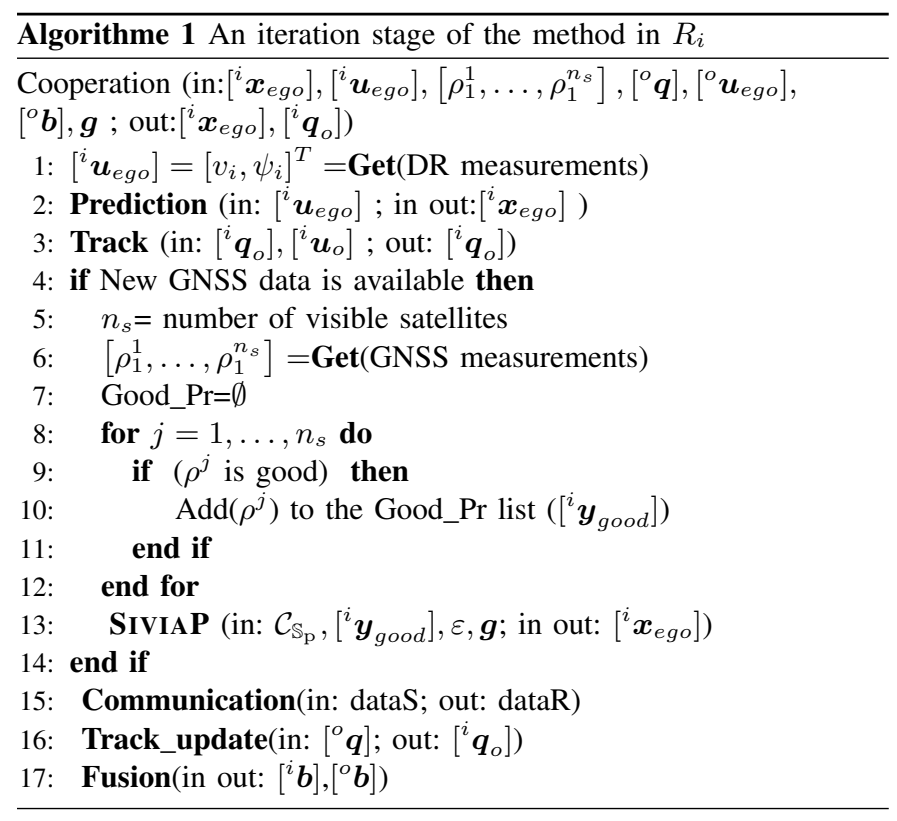

on the measurements of every satellite is performed. For every pseudorange measurement, we check if the $S N R$ (Signal to Noise Ratio) of the satellite is high enough (e.g. $35 \mathrm{~dB} / \mathrm{Hz}$ ) and we perform an innovation test based on a punctual estimate with the center of the boxes. Afterward, we apply the SIVIAP algorithm presented in [24] with the following modifications.

The considered solution is the hull box of $\overline{\mathbb{X}}$ which is the union of the inner $\mathbb{X}$ and the indeterminate $\Delta \mathbb{X}$ subpavings as it is shown in Fig 3 . In order to stop the bisections, we limit the computational time at $0.1 s$ for each epoch. In this case, the tolerable time communication delay is about 100 milliseconds.

In our problem, the vehicles have well synchronized clocks using PPS pulses of the GNSS receivers. The communication delays (line 15) are neglected.

The sent (dataS) and received (dataR) data at time instant $k$ by each vehicle have a unique identifier $i d$ in the group. The amount of the transmitted information on the communication network is low, since vehicles only exchange the lower and upper bounds of the boxes.

In line 16 of the algorithm, each vehicle $i$ updates the tracked position of the $R_{o}$ by the received estimated position $\left[{ }^{i} \boldsymbol{q}_{o}\right]:=\left[{ }^{o} \boldsymbol{q}_{\text {ego }}\right]$. Finally, $R_{i}$ merges its estimated biases with the received ones from the other vehicles $R_{o}$ as follows $\left[{ }^{i} \boldsymbol{b}\right]=\left[{ }^{i} \boldsymbol{b}\right] \cap\left[{ }^{o} \boldsymbol{b}\right]$.

\section{EXPERIMENTAL RESULTS}

The results of two scenarios, Standalone (S) and Cooperative (C), are reported to quantify the performance gain due to the cooperation using the proposed SIVIAP distributed method. We compare also the SIVIAP estimates with the ones of a rather conventional Bayesian procedure implementing an EKF [17] and involving the same processes: prediction/tracking, update, communication and fusion. The GNSS measurement errors are time correlated and, when doing sequential distributed estimation, the data fusion process that estimates the biases of these measurements incorporate loops. This induces a data incest problem. In the Bayesian framework, a usual method to address this issue is to do the fusion of the biases by using the Covariance Intersection (CI) operator, instead of the Simple Convex Combination (SCC) which is valid only when the errors are

\begin{tabular}{|c|c|c|}
\hline Variables & & Std. deviation \\
\hline \multirow{2}{*}{$\rho(m)$} & $\mathrm{R} 1$ & $\sqrt{90000.10^{-S N R / 10}}$ \\
\cline { 2 - 3 } & $\mathrm{R} 2$ & 9 \\
\hline \multirow{2}{*}{$v\left(\right.$ m.s $\left.^{-1}\right)$} & $\mathrm{R} 1$ & $1 e-3$ \\
\cline { 2 - 3 } & $\mathrm{R} 2$ & $2 e-3$ \\
\hline \multirow{2}{*}{$\psi\left(\right.$ rad.s $\left.^{-1}\right)$} & $\mathrm{R} 1$ & $2.5 e-3$ \\
\cline { 2 - 3 } & $\mathrm{R} 2$ & $5 e-3$ \\
\hline
\end{tabular}

Table I: Noises parameters used for the estimation methods.

uncorrelated. CI is known to provide consistent estimates even when facing an unknown degree of inter-estimate correlation [25], [26]. In the sequel, we denote by C-SIVIAP and C-EKF-CI the cooperative set-membership and Bayesian methods, respectively.

\section{A. Experimental setup}

The different approaches have been tested with two experimental vehicles (Fig. 2) and with the same data-set which was used in a post-processed way. A low-cost U-blox $4 T$ GPS receiver providing raw pseudoranges measurements at $5 \mathrm{~Hz}$ was used in each vehicle. The extraction of broadcast satellite navigation data has been done as follows. Conversion of U-blox navigation data into RINEX files and generation of satellite raw pseudoranges with ionosphere, troposphere, satellite clock offset and time relativity classical corrections. As the localization problem is studied in a local ENU (East-NorthUp) frame, the satellite positions, at their emission time, have be transformed into the ENU frame.

In every vehicle, a PolarX Septentrio receiver was used in RTK mode to provide ground truth data with heading $\psi$ information. Indeed, when the receiver is in motion, a GNSS receiver can calculate an accurate track angle which is the measured angle from true North in clock wise direction. When ground vehicles drive with low speed, one can assume that track is equal to heading since slippage can be neglected. A CAN-bus gateway was used to get the linear speed $v$ at $100 \mathrm{~Hz}$ rate. The inputs used by the cooperative system are $\boldsymbol{u}=\left[\begin{array}{ll}v & \psi\end{array}\right]^{T}$.

10 satellites were in view during the test and 5 of them were at least in common which satisfies the necessary condition of the observability study discussed in section II-B. When 4 common satellites between vehicles are not available, one should switch to standalone method. The GPS satellite visibility was sometimes very constrained due to buildings and trees near the test area. Vehicle $R_{2}$ has more satellite in view than vehicle $R_{1}$ during the experiment due to its favorable GNSS environments. The reported test was about 4 minutes long. Fig. 4 shows a top view of the trajectories of both vehicles using the (C) set-membership method.

The methods are compared with the same standard deviations parameters presented in Table I.

\section{B. SIVIAP performance analysis}

The distributed algorithm has been implemented in $\mathrm{C}++$ using the interval library IBEX [27] and with homemade functions. Fig. 4 shows the estimated position boxes of both vehicles. The displayed solution is the hull box $[\overline{\mathbb{X}}]$ of the union of the inner and indeterminate subpavings: $\underline{\mathbb{X}} \cup \Delta \mathbb{X}$.

Fig. 5 and 6 show the bounds of the position errors of the vehicles respectively for the $x$ and $y$ dimensions using the $(\mathrm{C})$ and $(\mathrm{S})$ methods. At a first glance, one can check the consistency of both observers due to the fact that bounds contain always the zero value. It means that the RTK reference position is always included in the estimated boxes which indicates a good tuning of the observers. 


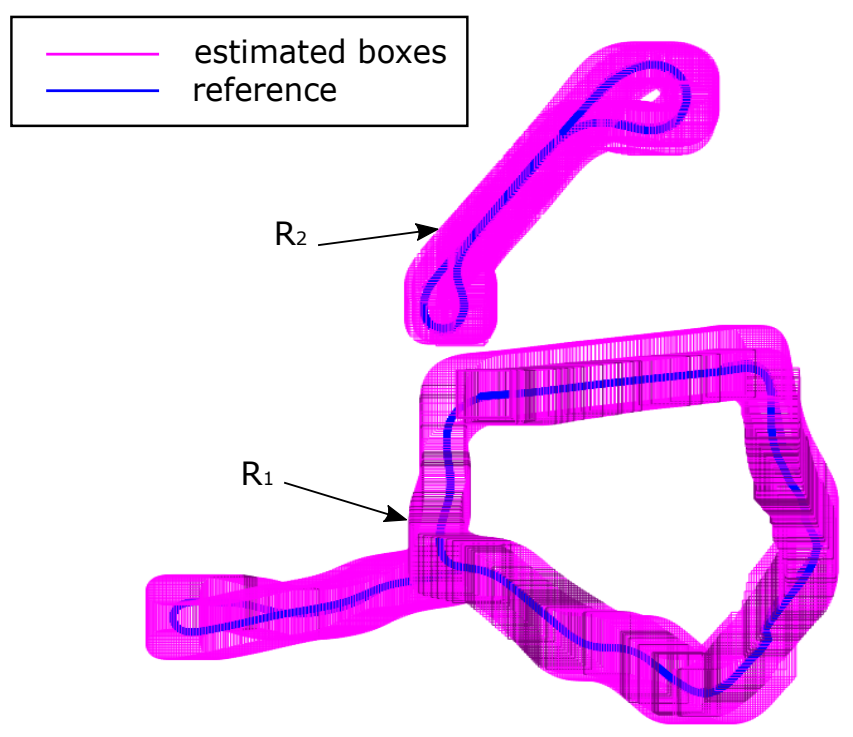

FIGURE 4: Trajectories of both vehicles when using C-SIVIAP. Reference and position boxes are displayed. The mean speed of vehicles was $30 \mathrm{Kmph}$. Every vehicle did several loops of its trajectory.
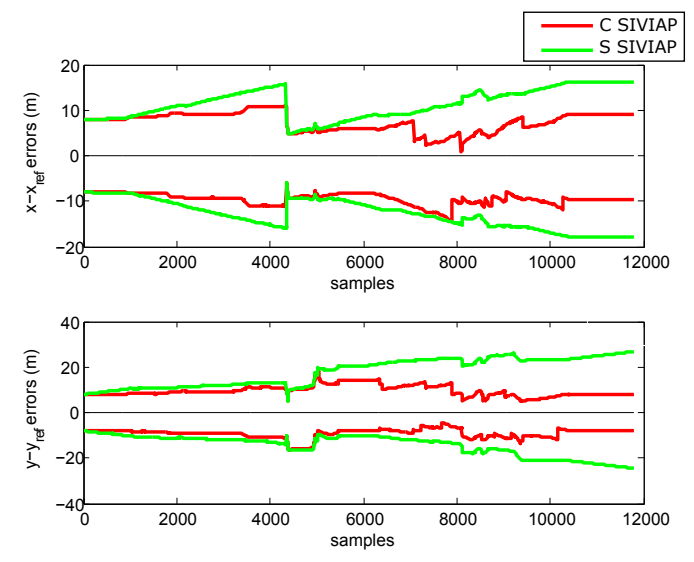

FIGURE 5: Bounds of position errors of $R_{1}$ centered on the reference.

Table II gives some performance metrics for both methods in term of absolute Horizontal Positioning Error (HPE) and relative distance. The Confidence Domain Size (CDS) of the resulting box is also studied, it is computed with the box volume. The CDS is evaluated via the Cumulative Distribution Function (CDF) of vehicles $2 D$ boxes volumes throughout the trajectory. A net improvement is obtained for vehicle $R_{1}$ in terms of accuracy and confidence. For instance, the median of HPE is reduced from $1.43 \mathrm{~m}$ to $0.89 \mathrm{~m}$ and the CDS is $66.4 \%$ condensed due to the cooperation since the $95 t h$ percentile of the CDS is less than $475.3 \mathrm{~m}^{2}$ compared to $1652 \mathrm{~m}^{2}$ when using the $\mathrm{S}$ method. Concerning vehicle $R_{2}$, the improvement of HPE is not as significant as in $R_{1}$ since contraction of boxes can move away the center from the reference. The cumulative CDS is $52.7 \%$ reduced since $95 \mathrm{th}$ percentile of the CDS is less than $256 \mathrm{~m}^{2}$ compared to $541.4 \mathrm{~m}^{2}$ when using the $\mathrm{S}$ method, which is a substantial improvement.

Regarding the estimation of the relative distance, the method improves the accuracy again trough the fusion of the biases estimates. Especially, if we look at the median and standard deviation errors, they are reduced by $42 \%$ and $20 \%$ respectively which is a significant improvement.
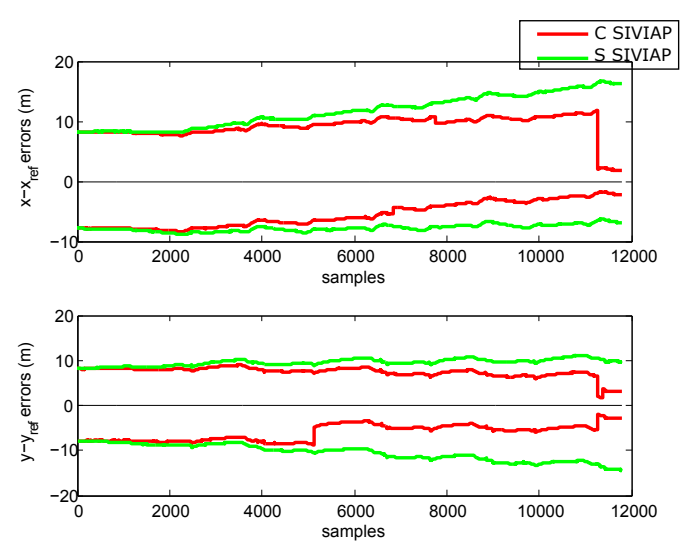

FIGURE 6: Bounds of position errors of $R_{2}$ centered on the reference.

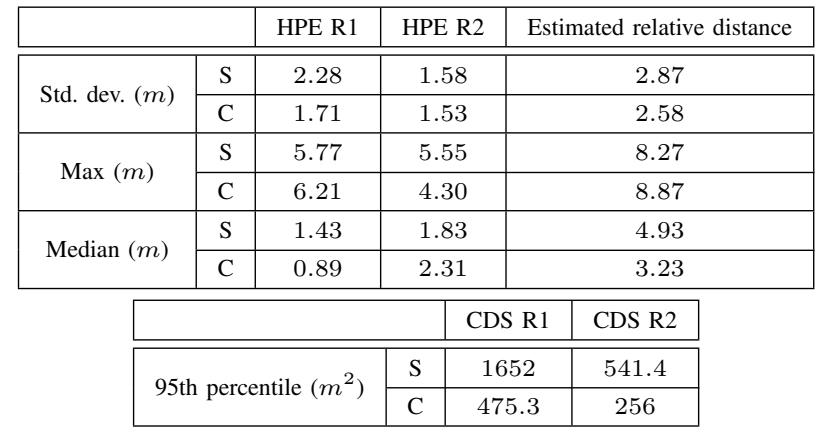

Table II: HPE, relative distance and CDS statistics.

The bias on every pseudorange has been initialized with the interval $[-30,30]$ (in meters) giving no prior knowledge. For each subplot of Fig. 7, the $x$ axis expresses the number of samples, the $y$ axis displays every estimate (the center of the box) of the bias $\left[b^{j}\right]$ with its bounds in meters and $j=1, \ldots, n_{s}$. Note that all subplots are truncated in order to observe the convergence illustrated by the horizontal final asymptotes. This convergence confirms the observability analysis. The obtained final values of the biases are very common for low cost GNSS receivers [28].

Other results illustrating more in details the behavior of the proposed
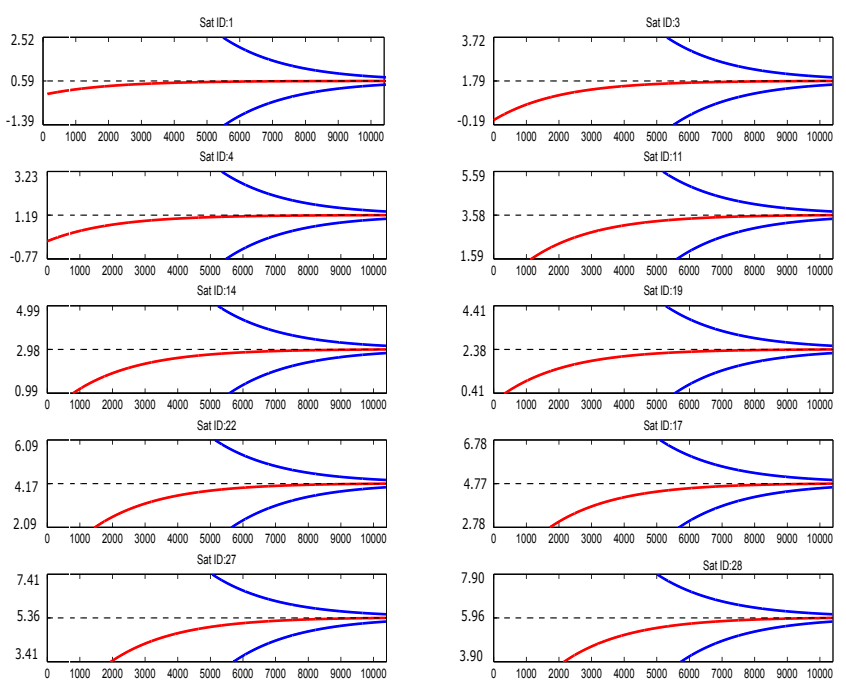

FIGURE 7: Estimates and bounds of pseudoranges biases (units are samples and meters). 
C-SIVIAP method can be found in [29].

\section{COMPARISON WITH DISTRIBUTED KALMAN FILTERING}

The cooperative estimation of the biases has been implemented with the CI data fusion operator [30]. Eq. (16) gives how the covariance matrix and the state estimate are computed.

$$
\left\{\begin{array}{c}
P^{-1}=\omega P_{1}^{-1}+(1-\omega) P_{2}^{-1} \\
\hat{x}=P \cdot\left(\omega P_{1}^{-1} \cdot x_{1}+(1-\omega) P_{2}^{-1} \cdot x_{2}\right)
\end{array}\right.
$$

The weighting factor $\omega \in[0,1]$ has been chosen here to minimize the determinant of the covariance matrix in order to get the smallest uncertainty. The CI fusion provides a conservative and robust result when correlation between two estimates $\hat{x}_{1}$ and $\hat{x}_{2}$ is unknown, as often the case in distributed systems. However, the data fusion algorithm is not optimal [31].

When using a cooperative Bayesian method based on Kalman filtering, vehicles have to share also their estimated error covariance matrix.

The C-EKF-CI has been implemented with exactly the same data as the C-SIVIAP method.

\section{A. Comparison criteria}

The choice of good performance metrics is a key issue for assessing a system and evaluating its application. For vehicle localization systems, accuracy is an important metric which usually refers to statistical figures of merit of the position error. These errors are built with respect to ground truth. For instance, the $95^{\text {th }}$ percentile of the horizontal positioning error distribution can be chosen for accessing the horizontal positioning accuracy. We also propose to evaluate the system reliability by examining the consistencies of the filters. This can be achieved by checking whether the estimated uncertainties correspond to the physical reality of the errors. The confidence bounds of estimated do mains also act as decision variables and so are linked to the pessimism of a localization system. If the confidence bounds are small while keeping the estimates consistent, the localization system is considered to be not too pessimistic. So, a good localization system is a system that provides adequate confidence information and good HPE accuracy. In the following, we use two criteria.

1) HPE: The HPE of both methods are defined below:

$$
H P E=\sqrt{e_{x}^{2}+e_{y}^{2}}
$$

where $e_{x}=\widehat{x}-x_{r e f}, e_{y}=\widehat{y}-y_{r e f} \cdot(\widehat{x}, \widehat{y})$ and $\left(x_{r e f}, y_{r e f}\right)$ represent respectively the $2 D$ estimated position and the RTK reference.

For the C-SIVIAP method, the center of the estimated hull box $\left(x_{m i d}, y_{m i d}\right)$ is used as a punctual estimate.

2) $C D S$ : The CDS needs to be assessed to check if the uncertainty is well handled. To gauge this issue, the $2 \mathrm{D}$ Cartesian evaluation is transformed in 1D problem by using a statistical distance computation denoted $k \sigma_{H P E}$, where $k$ is the chosen consistency risk according to a $\chi^{2}$ distribution (for a $10^{-2}$ risk, $k=3.035$ - this is a common choice that is done here). The $\sigma_{H P E}$ of a Bayesian method is given by Eq. 18 [32].

$$
\sigma_{H P E}=\sqrt{\frac{1}{\boldsymbol{u}_{e}^{T} P_{H P E}^{-1} \boldsymbol{u}_{e}}}, \text { with } \boldsymbol{u}_{e}=\left(\begin{array}{c}
e_{x} \\
e_{y}
\end{array}\right) / \sqrt{e_{x}^{2}+e_{y}^{2}}
$$

$\boldsymbol{u}_{e}$ is the unit vector supporting the HPE and $P_{H P E}$ is the estimated matrix of the error covariance when using C-EKF-CI (see Fig. 8 for an illustration).

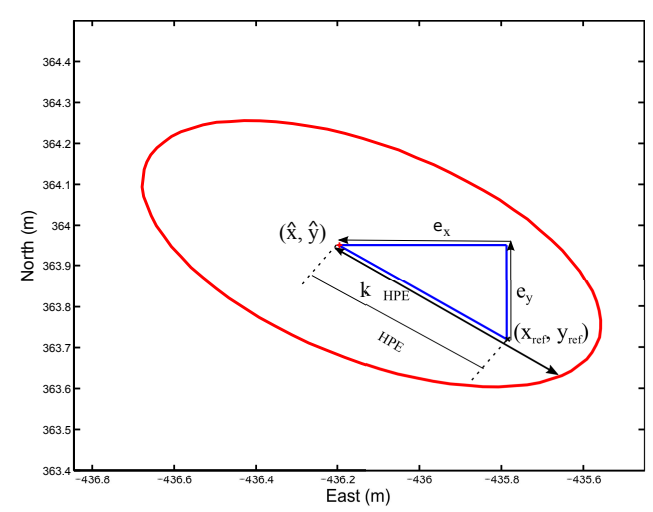

FigURE 8: $H P E$ and $C D S$ illustration for $C-E K F-C I$.

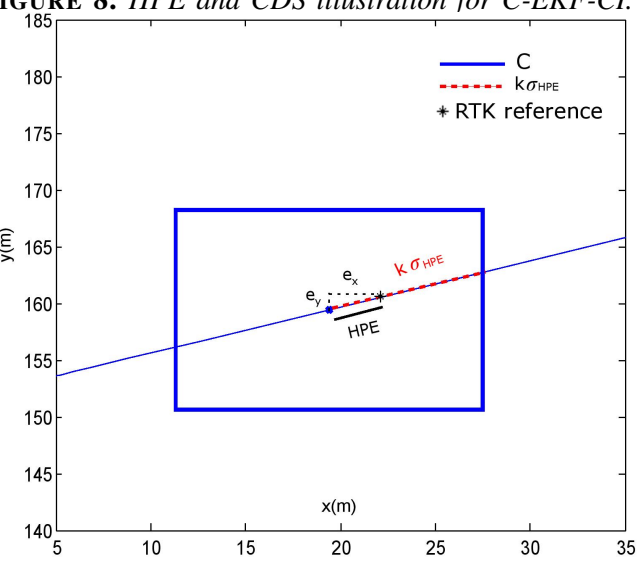

FIGURE 9: HPE and $k \sigma_{H P E}$ illustration for C-SIVIAP.

For the set-membership C-SIVIAP method, the same consistency $1 \%$ risk $k$ has been considered when setting the bounds on the pseudoranges:

$$
\left[\rho_{i}^{j}\right]=\left[\rho_{i}^{j}-k \sigma_{\rho}, \rho_{i}^{j}+k \sigma_{\rho}\right]
$$

where $j$ represents the satellite index and $\sigma_{\rho}$ represents the standard deviation of the pseudorange measurement presented in table I.

In way similar to the Bayesian method, the CDS of C-SIVIAP is defined by a $k \sigma_{H P E}$ segment in the direction of the HPE vector, as shown by figure 9 .

\section{B. Accuracy analysis}

Figure 10 depicts the cumulative distribution of HPEs of the both aforementioned methods. $95 \%$ of the HPE errors for vehicle $R_{1}$ are less than $10.46 \mathrm{~m}$ and $5.32 \mathrm{~m}$, respectively for C-EKF-CI and CSIVIAP. For vehicle $R_{2}$ these figures are less than $7.64 \mathrm{~m}$ and $4.41 \mathrm{~m}$ respectively. The accuracy gain of C-SIVIAP compared to C-EKF-CI is $49.13 \%$ for $R_{1}$ and $42.27 \%$ for $R_{2}$. The set-membership approach is clearly more accurate than the Bayesian one.

\section{Consistency analysis}

Let us consider now the filters consistency which is checked if the CDS bounds actually the HPE i.e. $H P E<k \sigma_{H P E}$. Consistency tests using HPEs are fundamental to provide suitable horizontal protection levels (HPL) for cooperative vehicles [33].

Figure 11 presents $2 D$ histograms in order to evaluate the consistency of the two methods for vehicles $R_{1}$ and $R_{2}$. The horizontal and vertical axis represent respectively the HPE and the CDS (i.e. $k \sigma_{H P E}$ ) 


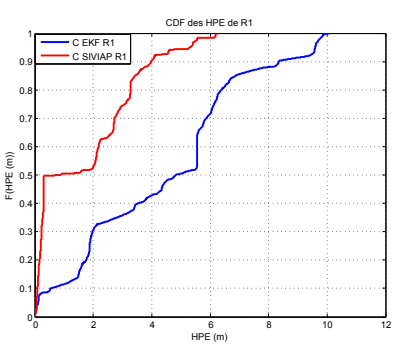

(a) Cumulative HPE of $R_{1}$.

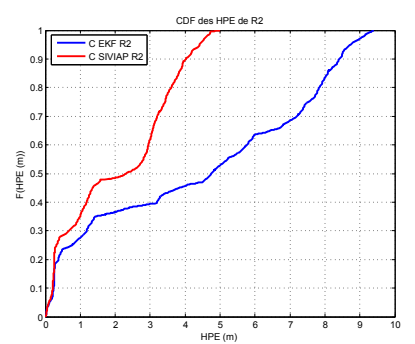

(b) Cumulative HPE of $R_{2}$.

Figure 10: Plots of the cumulative distribution function (CDF) of the HPE produced by the two methods in the two vehicles.

computed for each navigation solution. Each pixel tabulates the total number of occurrences of a specific (HPE, CDS) pair. Note that the color scale is logarithmic. These histograms can be considered as simplified Stanford diagrams since we are only interested in regions where we have CDS $>\mathrm{HPE}$ and HPE $>\mathrm{CDS}$. Points where the CDS is under the HPE error indicate a failure of integrity. In this way, the gray area corresponds to overconfident outcomes of the filters.

It appears from these results that the two methods are $100 \%$ consistent since there is no point in the gray area. Therefore, C-EKF-CI and C-SIVIAP methods are both reliable in the sense that the ground truth is always included in the estimated confidence domain.

As confidence is in practice compared to a threshold to indicate "use" or "don't use" to the client application, it is important, in terms of availability of the positioning information, to provide as small as possible confidence domains. Let us look especially at the $k \sigma_{H P E}$ of both methods for each vehicle in figure 11 .

It can be observed that the confidence domains produced by the $\mathrm{C}$ SIVIAP method are significantly tighter than the C-EKF-CI ones, in particular for vehicle $R_{2}$.

This indicates that the bounded-error method significantly reduces uncertainties compared to a Bayesian method based on covariance intersection fusion.

To resume the comparison analysis of the methods, the consistencies of set-membership and Bayesian filters are achieved. Both methods provide reliable confidence domains that contain the true positions of vehicles. Reliability is quite important for navigation missions in approach in order to avoid collision problem. However, the cooperative set-membership method perform better than the Bayesian one in terms of accuracy and uncertainty as it gives a significant improvement of positioning accuracy and a good decrease of the confidence domains.

If we look at performance comparison between the two vehicles when using the set-membership method, it can be observed that the best performance is obtained for vehicle $R_{2}$ which has more accurate positions (i.e, lower HPE) and less uncertainties (i.e, smaller CDS). This is due to the fact that $R_{1}$ has less satellites in view and its DR sensors are of less good quality compared to vehicle $R_{2}$.

\section{CONCLUSION}

This work has presented a cooperative localization technique for intelligent vehicles sharing GNSS common errors. It has been found that at least 4 satellites and an auto-regressive model of the biases are needed to keep the states observable which means that the problem is solvable even if there is no fixed base station well located.

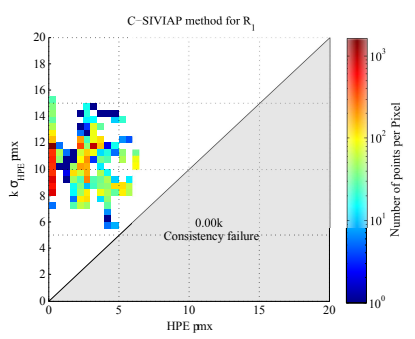

(a) C-SIVIAP for $R_{1}$.

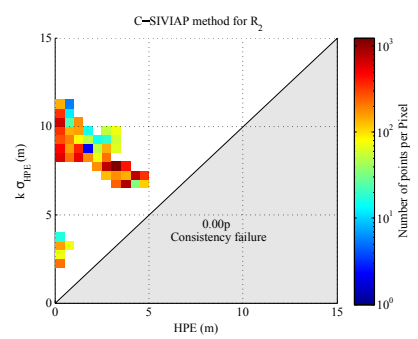

(c) C-SIVIAP for $R_{2}$.

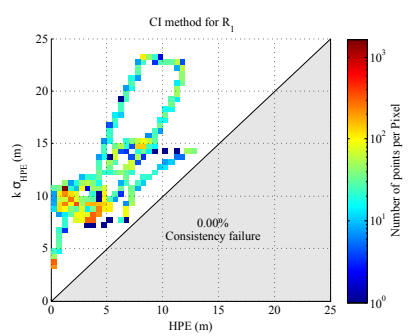

(b) C-EKF-CI for $R_{1}$.

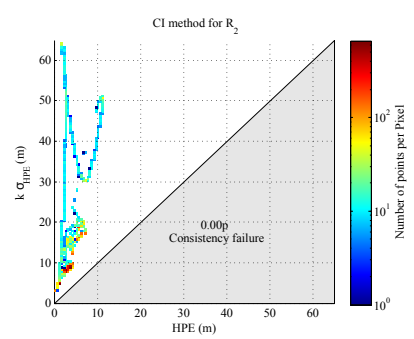

(d) C-EKF-CI for $R_{2}$.
FIGURE 11: simplified Stanford Diagram : Filters consistency when using cooperative set-membership and Bayesian methods for $R_{1}$ and $R_{2}$. (a) C-SIVIAP for $R_{1}$, (b) $C$-EKF based on CI fusion for $R_{1}$, (c) C-SIVIAP for $R_{2}$, (d) $C$-EKF based on CI fusion for $R_{2}$.

The proposed cooperative bounded error approach based on set inversion method with constraint propagation provides a significant enhancement in terms of accuracy and confidence domains compared to usual standalone methods.

The reuse of identical information (which are here the estimated biases) in the fusion process is also naturally managed by a setmembership approach. Such kind of method therefore deals correctly with the data incest issue. Moreover, it handles rigorously the nonlinearities of the equations. We have also reported a performance comparison of the interval method with a cooperative sequential Bayesian approach based on Kalman filtering and on covariance intersection fusion of the biases estimates. The experimental results indicate that both methods give reliable confidence domains of vehicles positions. However, the set-membership approach has the advantage to provide more accurate positions with smaller confidence domains. The key information deduced from this comparison is that set-membership methods are very suitable for applications requiring high integrity/accuracy in cooperative navigation contexts.

In future work, the robustness of the tuning of the proposed method should be more deeply considered by conducting different tests. This is important to address complex situations as urban canyons where simultaneous multipath issues are frequent. Moreover, the proposed algorithm has been designed to have a period higher that the communication delay between vehicles. In a V2X network, the transmission of real-time data with a guaranteed latency is not possible (due to collision issues for instance). In this case, a solution is to keep a time-stamped data buffer in each vehicle and to process the data in a sliding time window after the received data have been placed at the correct place in the buffer. The position is updated in the past and propagated up to the current time.

\section{Acknowledgment}

This work was carried out within the framework of the Equipex ROBOTEX (ANR-10- EQPX-44-01) and the Labex MS2T (ANR11-IDEX-0004-02). 


\section{REFERENCES}

[1] N. Karam, F. Chausse, R. Aufrere, and R. Chapuis. Localization of a group of communicating vehicles by state exchange. In IEEE Int Conf on Intelligent Robots and Systems, pages 519-524, Oct 2006.

[2] S. McLaughlin, V. Krishnamurthy, and S. Challa. Managing data incest in a distributed sensor network. In IEEE International Conference on Acoustics, Speech, and Signal Processing, volume 5, pages V-269-72 vol.5, April 2003.

[3] J. Monaco D. Ward and M. Bodson. Development and flight testing of a parameter identification algorithm for reconfigurable control. Journal of Guidance Navigation and Control, 21:948-956, 1998.

[4] L. Jaulin. A nonlinear set membership approach for the localization and map building of underwater robots. IEEE Trans. on Robotics, 25(1):8898, Feb 2009.

[5] Michel Kieffer and Eric Walter. Interval analysis for guaranteed nonlinear parameter and state estimation. Mathematical and Computer Modelling of Dynamical Systems, 11(2):171-181, 2005.

[6] Jaulin L. and Walter E. Set inversion via interval analysis for nonlinear bounded-error estimation. Automatica, 29(4):1053-1064, 1993.

[7] V. Drevelle and Ph. Bonnifait. Reliable positioning domain computation for urban navigation. IEEE Intelligent Transportation Systems Magazine, 5(3):21-29, 2013

[8] D. Meizel, O. Leveque, L. Jaulin, and E. Walter. Initial localization by set inversion. IEEE Trans on Robotics and Automation, 18(6):966-971, 2002.

[9] L. Jaulin. Interval constraint propagation with application to boundederror estimation. Automatica, 36(10):1547-1552, 2000.

[10] A. Bethencourt. Interval Analysis for swarm localization. Application to underwater robotics. PhD thesis, University of Bretagne, Sept 2014.

[11] L. Conde, R. Chelim, and U. Nunes. Collaborative vehicle selflocalization using multi-gnss receivers and $\mathrm{v} 2 \mathrm{v} / \mathrm{v} 2 \mathrm{i}$ communications. In IEEE 18th Int Conf on Intelligent Transportation Systems, pages 25252532, Sept 2015.

[12] Kyoung-Hwan Jo and Jihong Lee. Cooperative localization of multiple robots with constraint propagation technique. In IEEE Int Conf on Intelligent Robots and Systems, pages 3477-3482, Sept 2008.

[13] E. Richter, M. Obst, R. Schubert, and G. Wanielik. Cooperative relative localization using vehicle-to-vehicle communications. In International Conference on Information Fusion, pages 126-131, July 2009.

[14] N. Mattern, M. Obst, R. Schubert, and G. Wanielik. Cooperative vehicle localization algorithm - evaluation of the covel approach. In International Multi-Conference on Systems, Signals and Devices (SSD), pages 1-5, March 2012.

[15] U. Bhatti, W. Ochieng, and S. Feng. Integrity of an integrated gps/ins system in the presence of slowly growing errors. GPS Solutions, (3):73181, 2007.

[16] E. D. Kaplan and C. Hegarty. Understanding GPS: Principles and Applications. Second Edition, Artech House, 2005.

[17] K. Lassoued, I. Fantoni, and P. Bonnifait. Mutual localization and positioning of vehicles sharing GNSS pseudoranges: Sequential bayesian approach and experiments. In IEEE Int Conf on Intelligent Transportation Systems, Spain, Sept 2015.

[18] X. Xiao J. Rife. Estimation of spatially correlated errors in vehicular collaborative navigation with shared GNSS and road-boundary measurements. In Int Technical Meeting of The Satellite Division of the Institute of Navigation, pages 1667-1677, Sept 2010.

[19] R. Hermann and A.J. Krener. Nonlinear controllability and observability. IEEE Trans on Automatic Control, 22(5):728-740, Oct 1977.

[20] A. Martinelli and R. Siegwart. Observability analysis for mobile robot localization. In IEEE Int Conf on Intelligent Robots and Systems, pages 1471-1476, Aug 2005.

[21] X.S. Zhou and S.I. Roumeliotis. Robot-to-robot relative pose estimation from range measurements. IEEE Trans on Robotics, 24(6):1379-1393, Dec 2008.

[22] D. Waltz. Generating semantic descriptions from drawings of scenes with shadows. The psychology of computer vision, 1975.

[23] K. Lassoued, O. Stanoi, Ph. Bonnifait, and I. Fantoni. Mobile robots cooperation with biased exteroceptive measurements. In Int Conf on Control Automation Robotics Vision, pages 1835-1840, Singapore, Dec 2014.

[24] L. Jaulin, M. Kieffer, O. Didrit, and E. Walter. Applied interval analysis with examples in parameter and state estimation, robust control and robotics. Springer, 2001.
[25] H. Li and F. Nashashibi. Cooperative multi-vehicle localization using split covariance intersection filter. IEEE Intelligent Transportation Systems Magazine, 5(2):33-44, Summer 2013.

[26] CY. Chong and S. Mori. Convex combination and covariance intersection algorithms in distributed fusion. In 4th International Conference on Information Fusion, Canada, 2001.

[27] G. Chabert. IBEX(http://www.ibex-lib.org), 2007.

[28] O. Le Marchand, Ph. Bonnifait, J. Ibanez-Guzman, D. Betaille, and F. Peyret. Characterization of gps multipath for passenger vehicles across urban environments. ATTI dell'Istituto Italiano di Navigazione, (189):77-88, 072009.

[29] K. Lassoued, P. Bonnifait, and I. Fantoni. Cooperative localization of vehicles sharing gnss pseudoranges corrections with no base station using set inversion. In IEEE Intelligent Vehicles Symposium (IV), Gothenburg, Sweden, June 19-22 2016.

[30] N. R .Ahmed, S. J . Julier, J. R . Schoenberg, and M. E . Campbell. Multisensor Data Fusion From Algorithms and Architectural Design to Applications, chapter Decentralized Bayesian Fusion in Networks with Non-Gaussian Uncertainties, pages 383-408. CRC Press, 2015.

[31] U. W. Utete. Network Management in Decentralized Sensing Systems. $\mathrm{PhD}$ thesis, Department of Engineering Science, University of Oxford, 1995.

[32] V. Drevelle and Ph. Bonnifait. Localization confidence domains via set inversion on short-term trajectory. IEEE Transactions on Robotics, 29(5):1244-1256, 2013.

[33] M. Worner, F. Schuster, F. Dolitzscher, C. G. Keller, M. Haueis, and K. Dietmayer. Integrity for autonomous driving: A survey. In IEEE/ION Position, Location and Navigation Symposium (PLANS), pages 666-671, April 2016.

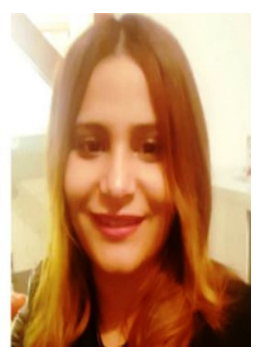

Khaoula Lassoued graduated as an automatic electric engineer from the ENIG School in Tunisia, then she succeeded in a research MASTER thesis in the DRIVE laboratory of the University of Burgundy in France. She obtained her Ph.D. degree in Computer Science and Engineering at the Heudiasyc laboratory, at the Université de Technologie de Compiègne (UTC) member of Sorbonne University, France in 2016. Her topic of research is the navigation of heterogeneous mobile robotic systems in mutual cooperation.

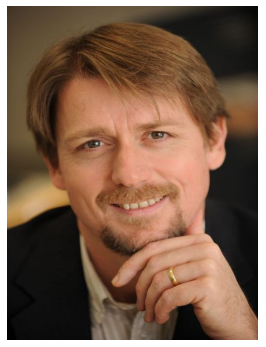

Philippe Bonnifait is a professor in the Computer Science department of the Université de Technologie de Compiègne (UTC), France. He obtained his Ph.D. degree in automatic control and computer science from the École Centrale de Nantes in 1997. Since 1998, he has been with Heudiasyc UMR 7253, a common research laboratory between UTC and CNRS. His research interests are in Intelligent Vehicles and Robotics. He is currently working on high integrity positioning methods for autonomous systems and self-driving vehicles. 


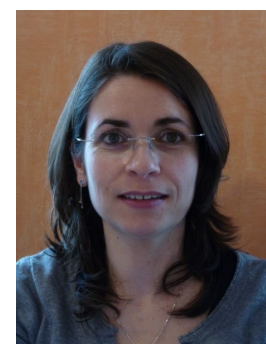

Isabelle Fantoni received the $\mathrm{PhD}$ degree in Nonlinear Control for Underactuated Mechanical Systems, in 2000 from the Université de Technologie de Compiègne, France.

Since October 2001, she is a CNRS Researcher at Heudiasyc laboratory, in Compiègne, France, and Research Director since October 2013. Her research interests include non-linear control, Unmanned Aerial Vehicles, fault-tolerant control for UAVs, heterogeneous robotic systems in cooperation. 